\title{
Ophthalmologica
}

Ophthalmologica 2016;235:241

DOI: 10.1159/000444811
Received: February 9, 2016

Accepted: February 16, 2016

Published online: March 10, 2016

\section{Surgical Management of a Case of a 360-Degree Giant Retinal Break}

\author{
Ahmed Elshewy \\ Ophthalmology Department, Faculty of Medicine Kasr Al Ainy, Cairo University Hospital, Cairo, Egypt
}

\begin{abstract}
Purpose: The aim is to describe tools and techniques that can be used to deal with total retinal detachment (open funnel) and associated proliferative vitreoretinopathy due to a 360-degree giant retinal break following ocular trauma. Setting/Venue: The video (available at www. karger.com/doi/10.1159/000444811) was created at the Ophthalmology Department, Faculty of Medicine Kasr Al Ainy, Cairo University Hospital, Cairo, Egypt. Methods: A 16-year-old female patient presented to the Cairo University Hospital after blunt ocular trauma. She had traumatic cataract, and ultrasound examination showed total retinal detachment. She was scheduled for surgery at the Cairo University Hospital. Phacoemulsification of traumatic cataract was done after insertion of the 3-port 23-gauge trocar system. The 25-gauge chandelier illumination system was used to assist the peeling of adherent posterior hyaloid and epiretinal membranes, allowing bimanual work using an end-gripping forceps and a diamond-dusted retinal scrapper. Removal of the vitreous surrounding the retinal funnel
\end{abstract}

was performed, and a small perfluorocarbon (PFC) bubble was used to support the posterior pole. This was followed by refreshing the rolled edges of the retinal break and shaving the vitreous base and anterior leaflet of the giant break by surgeon-assisted scleral indentation. The eye was completely filled with PFC, and Argon laser retinopexy was performed. Direct PFC-silicone oil exchange was done to avoid retinal slippage (silicone oil 5,000 cSt was used). Results: Retinal attachment was successfully achieved at the end of the surgery. The silicone oil was removed 5 months after the initial surgery. The patient's uncorrected visual acuity 1 month after removal of the silicone was 0.4. Conclusions: The chandelier-assisted bimanual technique is an effective method for dealing with adherent posterior hyaloid and epiretinal membranes in a detached retina, particularly in cases of complex open-funnel retinal detachment. Proper shaving of the anterior leaflet and refreshing the edges of the retinal break helps decrease postoperative PVR formation. Direct PFC-silicone oil exchange in giant retinal breaks helps minimize the risk of retinal slippage occurrence.

(c) 2016 S. Karger AG, Basel

\section{KARGER}

E-Mail karger@karger.com www.karger.com/oph
C 2016 S. Karger AG, Basel

0030-3755/16/2354-0241\$39.50/0
Ahmed Elshewy

Ophthalmology Department, Faculty of Medicine Kasr Al Ainy

Cairo University Hospital, Kasr Al Ainy Street

EG-11562 Cairo (Egypt)

E-Mail ahmshewy@gmail.com 